\title{
Epidemiology of Group B Streptococcus in Saudi Parturient Women in a Private Hospital
}

\author{
Tasnim Zahid Hussain \\ Kingdom Hospital and Consulting Clinics, Riyadh, Saudi Arabia \\ Correspondence should be addressed to: Tasnim Zahid Hussain ; misstahir@doctor.com \\ Received date: 28 May 2014; Accepted date: 5 August 2014; Published date: 23 March 2015 \\ Academic Editor: Rooyen Tinago Mavenyengwa
}

Copyright (C) 2015. Tasnim Zahid Hussain. Distributed under Creative Commons CC-BY 4.0

\begin{abstract}
Objective: Maternal colonization with group B streptococcus (GBS), during pregnancy, in the private medical sector has not been studied thus far and the objective of this study was to gather relevant data in the private medical sector. Methods: A retrospective, crosssectional, review of GBS colonization among all patients who delivered at Kingdom Hospital, Riyadh, Saudi Arabia, between January, 2007 and December, 2010 was performed. Data on colonization rate, complications, antibiotics coverage and neonatal outcome were collected. Results: During the four year interval 246 women out of 3253 had antenatal GBS colonization (7.6\%). Antenatal urinary tract infection was present in $55(22.4 \%)$ and prelabour rupture of membranes in $65(26.4 \%)$ of total 246 colonized women. There were no cases of maternal endometritis, septicemia or neonatal early-onset GBS disease. Intrapartum prophylactic antibiotic coverage was provided to 191 of 237 (80.6\%) colonized women. Conclusion: Our results support that GBS colonization in the private medical sector in Saudi Arabia is less than that reported from the public medical sector. Antenatal urinary tract infection and pre-labour rupture of membranes are common co morbidities. We suggest a prospective randomized trial comparing public and private medical sector patients for the GBS colonization rate, risk factors for colonization and complications, along with long term health effects of antibiotics use. It will aid us to implement new protocols for GBS positive women.
\end{abstract}

Keywords: Group B Streptococcus, colonization, labour, early-onset neonatal disease

\section{Introduction}

In 1938, Streptococcus agalactiae, or group B streptococcus (GBS), was identified as a human pathogen with a considerable impact on public health (Fry, 1938). Obstetric consequences were initially reported as ranging from silent carriage in the vagina of asymptomatic parturient women (Lancefield and Hare, 1935) to being the leading cause of fatal puerperal sepsis (Fry, 1938). By 1970, GBS was also identified as a causative agent in meningitis and septicemia in neonates and infants $<3$ months of age, as mentioned in the book by Mandell et al. (2009).

Cite this Article as: Tasnim Zahid Hussain (2015), "Epidemiology of Group B Streptococcus in Saudi Parturient Women in a Private Hospital" Obstetrics \& Gynecology: An International Journal, Vol. 2015 (2015), Article ID 376062, DOI: 10.5171/2014.376062 
GBS is a gram-positive, beta hemolytic diplococcus (Mandell et al. 2009) that resides in the genital tract or lower gastrointestinal tract of up to $30 \%$ of women (ACOG, 2011). Factors that have been linked to GBS colonization in women include race, being more common in black women (Newton et al. 1996); sexual activity, particularly oral sex (Manning et al. 2002); and conditions such as diabetes (Mandell et al. 2009) or carbohydrate intolerance in pregnancy (Ramos et al. 1997). There are, however, case reports of GBS colonization among sexually inactive persons, as proposed in a research article by Manning et al. (2004).

In the book by Mandell et al. (2009), it is stated that the detection rate of GBS is influenced by the site sampled and detection method. Higher detection rates have been reported using multiple sampling sites such as lower vagina and rectum. In addition, the use of selective broth medium increases the detection rate by $20 \%$.

GBS is known for early and late-onset neonatal infection, which results from the passage of the previously unaffected fetus through the colonized maternal birth canal (Mandell et al. 2009). ACOG (2011) has proposed the risk factors that predispose an infant from a colonized mother, to neonatal GBS infection and these are preterm delivery ( $<37$ weeks of gestation), prolonged rupture of membranes $(>18$ hours), intra-amniotic infection (reflected by fever $>38^{\circ} \mathrm{C}$ ), young maternal age, black race, and history of a previous GBS-infected child. It has been shown by Winn (2007), that in the absence of any treatment, neonatal colonization rates are as high as $75 \%$, among who up to $1 \%$ to $2 \%$ will develop early onset neonatal GBS disease. The case fatality of early-onset neonatal GBS infection can reach 50\% (Winn, 2007), and for late-onset neonatal disease is reported at 3\% to 5\% (Mandell et al. 2009). Maternal consequences of GBS infection can include urinary tract infection, prelabour rupture of membranes (PROM) with its related prematurity, endometritis, septicemia (Mandell et al. 2009) and, rarely, meningitis (ACOG, 2011).
Boyer and Gotoff (1986) reported that intrapartum antibiotic prophylaxis (IAP) to colonized mothers can significantly prevent early-onset neonatal GBS disease. This discovery was acknowledged worldwide and led to the implementation of screening prenatal women near term, and giving intrapartum penicillin to women with positive GBS cultures (ACOG, 2011). Adoption of this practice led to a marked reduction in cases of early-onset neonatal GBS infection (Mandell et al. 2009).

Regional carriage rates for GBS in pregnant women, ranging from $8 \%$ to $18 \%$, have been reported in a research study by Stoll and Schuchat (1998); for example, prevalence in India/Pakistan 9\%, Asia/Pacific 8\%, Sub-Saharan Africa 18\%, Middle East/North Africa $17 \%$ and the Americas $12 \%$. Globally, the overall carriage rate is estimated at $13 \%$.

A U.S. study by Lewin and Amstey (1981) showed an antenatal GBS colonization rate of $19 \%$, in pregnant women attending private medical sector hospitals. Also, Hickman et al. (1999) from the United States compared the GBS colonization rate among women in the private and public medical sectors. They reported that maternal GBS colonization was similar in private $(25 \%)$ and public hospitals $(30 \%)$, and that maternal socioeconomic status had no influence on the colonization rate. The reported neonatal colonization rate was less at private $(4 \%)$ compared with public $(20 \%)$ sector hospitals. The authors linked this finding to more antibiotic use in the private hospitals (35\%), than in the public hospitals (17\%).

In another study by Faro et al. (2010) from the United States, focusing only on private medical sector patients, reported a $29 \%$ GBS colonization rate, with an incidence of neonatal GBS disease was 0.94 per 1000 live births. A study from Greece by Tsolia et al. (2008) compared antenatal GBS colonization between the private and public medical sector and found higher prevalence in the private medical sector $(10 \%$ vs. $3.9 \%)$, with an overall colonization rate of $6.6 \%$. They reported that significant higher colonization rate is 
associated with higher numbers of prenatal visits $(p=0.002)$.

Early reports from Saudi Arabia estimate a GBS colonization rate in pregnant women in the third trimester ranging from $9.2 \%$ to 27.6\% [Uduman et al. (1985), Al-Suleiman et al. (1991), Madani et al. (1998), El-Kersh et al. (2002)]. A recent study in a public hospital by Zamzami et al. (2011) reported a $31.6 \%$ GBS colonization rate in parturient women. Al-Muneef et al. (2000), in another study have reported the neonatal colonization rate in a public hospital in Saudi Arabia at 0.8/1000 live births.

We studied the prevalence of GBS colonization among pregnant women and subsequent neonatal outcomes in a private hospital in Riyadh, Saudi Arabia.

\section{Methods}

A retrospective, cross sectional, medical record review was performed for all women who presented in labour at Kingdom Hospital, a private hospital in Riyadh, Saudi Arabia, during the 4-year period from January 2007 through December 2010. The study was approved by the hospital ethics committee. All pregnant women booked at Kingdom Hospital were offered GBS screening at 3537 weeks, using anogenital swab (AGS). The organism was grown on blood agar culture medium, at $37^{\circ} \mathrm{C}$, for $48-72$ hours. Antenatal urine cultures were performed in women with suspected urinary tract infection due to any cause. GBS colonized women were informed about intrapartum antibiotic prophylaxis (IAP) and no repeat test was done at the time of labour.
Colonized women were given intravenous ampicillin in the active phase of labour, according to the hospital protocol.

The medical records of women who were colonized with GBS and their neonates were reviewed. Data acquired for pregnant women included parity, gestational age at the time of delivery, mode of delivery, premature rupture of membranes, other antenatal infections, site of GBS colonization, complications of GBS infection, and intrapartum prophylactic antibiotic coverage.

A protocol in the Kingdom Hospital nursery defines close observation of infants born to GBS colonized mothers, with further tests for infection performed if there is a suspicion of infection based on symptoms. These tests include a complete blood picture for raised WBC and blood cultures for GBS. Data acquired for babies born to mothers who were colonized with GBS included the outcome at birth and condition at the time of discharge and at the first presentation in the neonatal period due to any illness.

\section{Results}

During the study period, 5487 women were booked for antenatal care at Kingdom Hospital, of whom 3253 (59.3\%) were screened. GBS colonization was detected in $246(7.6 \%)$ women, who delivered 251 babies (5 twins) (Table 1). GBS was found in the AGS alone in 186 (75.6\%), urine in $28(11.4 \%)$ and both AGS and urine sample in $32(13 \%)$ women. (Table 2$)$

Table 1: Representing the year-based statistics of all patients.

\begin{tabular}{|c|c|c|c|c|c|}
\hline Year & Total Deliveries & Booked patients & GBS screened & GBS +ve (n) & Percentage \\
\hline 2007 & 1835 & 1515 & 817 & 52 & $6.4 \%$ \\
\hline 2008 & 1688 & 1230 & 683 & 61 & $8.9 \%$ \\
\hline 2009 & 1605 & 1330 & 915 & 56 & $6.1 \%$ \\
\hline 2010 & 1772 & 1412 & 838 & 77 & $9.1 \%$ \\
\hline Total & 6900 & 5487 & 3253 & 246 & $7.6 \%$ \\
\hline
\end{tabular}


Table 2: Further results of the study

\begin{tabular}{|c|c|c|c|c|}
\hline VARIABLES & \multicolumn{4}{|c|}{ DETAILS (n) } \\
\hline GBS Cases & \multicolumn{4}{|c|}{ Positive $246(7.6 \%)$} \\
\hline Colonization site (246) & \multicolumn{4}{|c|}{\begin{tabular}{l|l} 
& Urine $=28(11.48 \%)$ \\
\end{tabular}} \\
\hline Parity (246) & \multicolumn{2}{|c|}{ Primi $=73(29.7 \%)$} & \multicolumn{2}{|c|}{ Multi $=173(70.3 \%)$} \\
\hline Gestational age (246) & Term $=228(92.7 \%)$ & \multicolumn{2}{|c|}{ Post term $=1(0.4 \%)$} & Preterm = $17(6.9 \%)$ \\
\hline Maternal complications (246) & \multicolumn{2}{|c|}{ Antenatal UTI= $65(26.4 \%)$} & \multicolumn{2}{|c|}{ PPROM= $55(22.4 \%)$} \\
\hline Intrapartum Prophylaxis (237) & \multicolumn{2}{|c|}{ Given= $191(80.6 \%)$} & \multicolumn{2}{|c|}{ Missed $=46(19.4 \%)$} \\
\hline
\end{tabular}

The mode of delivery was elective cesarean for 9 women, (3.6\%), emergency cesarean for $40 \quad(16.3 \%)$, normal spontaneous vaginal delivery for 174 (70.7\%), and operative vaginal delivery for 23 women $(9.3 \%)$.

Fifty five (22.4\%) women had PROM, and $65(26.4 \%)$ had a urinary tract infection. Out of these 65 women with urinary infection, GBS was the sole causative agent in $56(86.2 \%)$, GBS with other coexisting organism, E. coli, Klebsiella pneumonia and MRSA, in $4(6.2 \%)$ and organisms other than GBS, E. coli, Klebsiella pneumonia and Kluyvera in $5(7.7 \%)$ women. There was no case of endometritis or sepsis.

Intrapartum prophylactic antibiotics were given to 191 of 237 (80.6\%) women who underwent vaginal delivery. 32 women (13.5\%) were given intravenous ampicillin post delivery.

Maternal colonization status was missed by the pediatrician in 57 cases $(22.7 \%)$, and records were not available for 9 cases (3.6\%). Blood tests were warranted in 82 of 185 (44.3\%) neonates for which data were available and who were managed according to the protocol, all of which were negative for GBS. There was no case of early-onset GBS disease among 251 babies.

In the early neonatal period, 25 babies presented at the Kingdom hospital for miscellaneous reasons. Of these, there were 15 cases of upper respiratory tract infection (60\%) and 9 of them were severe.

\section{Discussion}

The $7.6 \%$ prevalence of GBS colonization in our private hospital sample is lower than $9.2 \%$ (Uduman et al. 1985) to $31.6 \%$ (Zamzami et al. 2011) reported from public healthcare facilities in Saudi Arabia. Our rate is also low compared with colonization rates among private healthcare centers in other countries, ranging from $10 \%$ in Greece (Tsolia et al. 2003) to $29 \%$ in the US (Faro et al. 2010).

Our sample of 3,253 women screened is larger than previously reported from public hospitals in Saudi Arabia. Most studies included $<400$ women, with a study of 1939 women performed more than a decade ago (Al-Suleiman et al. 1991). We therefore present the most recent data on a larger sample size in a private hospital.

Lewin and Amstey (1981) reported that GBS is spontaneously lost during the course of pregnancy in 51\% women. Our study women were not screened again at the time of labour. The observation from the US study needs to be evaluated further by re-screening third trimester GBS positive women, at the time of labour. A significant reduction of GBS colonization at labour will reduce the number of women receiving IAP.

In our study, 85 women (44.5\%) were given intrapartum prophylactic antibiotics for $<4$ hours. There was no clinical neonatal infection. This is in contrast with the research published by Turrentine et al. (2013) in the US, which concludes that clinical neonatal sepsis is increased in 
mothers who receive $<4$ hours of antibiotic compared to $>4$ hours.

It has been proposed in many research studies that private sector hospitals are notorious in prescribing antibiotics [(Hickman et al. (1999), Basu et al. (2012), Sharma et al. (2012)]. In addition, it is known that the over-use of antibiotic leads to resistant strains of bacteria (Tacconelli et al. 2008). In our study, almost 1 in every 10 patients, was given antibiotic post delivery to which we could find no genuine indication. The guidelines need to be appropriately implemented, and regularly audited in the private hospitals to avoid unnecessary antibiotic use. We also recommend a prospective trial to review the long term health effect of IAP in colonized mothers and their off springs.

In the United Kingdom, the policy of routine screening of all pregnant women for GBS colonization is not implemented since 2008, because of concerns about anaphylaxis related to antibiotic use and the emergence of resistant bacteria (RCOG, 2012). However, the Royal College of Obstetricians and Gynecologists recommends giving intrapartum penicillin prophylaxis to women with specific risk factors. In our study, there was no neonatal transmission, even in GBS positive mothers who were not treated in labour incidentally. There is no debate on GBS being a fatal microorganism but do we need to risk all our patients' immunity with penicillin is a momentous question. We, therefore, suggest considering a shift towards risk-based approach rather than culture positive-approach for prescribing IAP.

The pre-labour rupture of membranes and urinary tract infection is common $(22.4 \%$ \& $26.4 \%$ respectively) co-morbidity in colonized mothers. GBS screening in third trimester cannot predict earlier urinary tract infection although such women may be considered at high risk of having PROM. We do not know if antenatal patients with urinary tract infection, by any microorganism, are at high risk of harboring GBS at term. And if we can prevent PROM and its associated morbidity in colonized mothers. Further research in this area is recommended.

The finding of more respiratory illnesses among babies after delivery needs to be evaluated further to confirm or refute any relation to maternal GBS colonization.

We, in conclusion, suggest a prospective randomized trial comparing GBS colonization in the public and private medical sector to determine the risk factors of antenatal GBS colonization, maternal and neonatal complications of GBS with focus on potential causes and long term effects of antibiotic use in mothers and their newborns. We also need to compare colonized mothers who are given intrapartum prophylactic antibiotics to those who do not receive any treatment. The resultant data will grant us a foundation, to suggest and implement new recommendations.

\section{Acknowledgement}

The author would like to thank Dr. Omar Hasan Kasule, who helped with the proposal and design of this study, the valued staff from Kingdom Hospital, Maysoon Amro and Sawsan Shawawreh, Head Nurse and Deputy Head nurse in the Labour Ward and Mr. Shariff Duruin, Medical Records Manager, for their generous support in the data collection and medical records review. Gratitude is extended to Dr. Valerie Zimmerman for her assistance in writing this article.

\section{References}

1. Al-Muneef, M, Alalola, S, Ahmed, S, Memish, Z, Khan, M. Y. and Al-Shaalan, M. (2000) "The changing spectrum of group B streptococcus (GBS) infection in infants of Saudi Arabia," Journal of chemotherapy, 12 (1) 48-52.

2. Al-Suleiman, S. A, Farrag, I, Kingsley, T. D, Uduman, S. A. and Al-Mouzan, M. I. (1991) "Third trimester colonization and treatment of group B beta hemolytic streptococcus among obstetric patients in the eastern province of Saudi Arabia," 
Journal of obstetrics and gynecology, 11 (6) 409-413.

3. American College of Obstetricians and Gynecologists. (2011) "Prevention of earlyonset group $B$ streptococcal disease in newborns" [Online] USA: ACOG Committee Opinion number 485. [Accessed July 30, 2014], Available at: http://www.acog.org/ /media/Committe e\%200pinions/Committee\%20on\%200bst etric\%20Practice/co485.pdf?dmc $=1 \&$ ts $=2$ $0140730 \mathrm{~T} 0837533686$

4. Basu, S, Andrews, J, Kishore, S, Panjabi, $\mathrm{R}$ and Stuckler, D. (2012) "Comparative performance of private and public healthcare systems in low- and middleincome countries: a systematic review," [Online]. PLos Medicine, [Accessed July 31, 2014], Available at: http://www.plosmedicine.org/article/info \%3Adoi\%2F10.1371\%2Fjournal.pmed.100 1244

5. Boyer, K. M. and Gotoff, S. P. (1986) "Prevention of early-onset neonatal group B streptococcal disease with selective intrapartum chemoprophylaxis," New England Journal of Medicine, 314 (26) 1665-1669.

6. El-Kersh, T. A, Al-Nuaim, L. A, Kharfy, T. A, Al-Shammary, F. J, Al-Saleh, S. S. and AlZamel, F. A. (2002) "Detection of genital colonization of group B streptococci during late pregnancy," Saudi Medical Journal, 23 (1) 56-61.

7. Faro, S, Brehm, B, Smith, F, Mouzoon, M, Greisinger, A, Wehmanen, 0 . and Turrentine, M. A. (2010) "Screening for group B streptococcus: a private hospital's experience," [Online] Infect Dis Obstet Gynecol, [Accessed July 31, 2014], http://www.hindawi.com/journals/idog/2 010/451096/

8. Fry, R. M. (1938) "Fatal infections by haemolytic streptococcus group B," Lancet, 1 199-201.
9. Hickman, M. E, Rench, M. A, Ferrieri, P. and Baker, C. J. (1999) "Changing epidemiology of group B streptococcal colonization," Pediatrics, 104 (2 pt 1) 203209.

10.Lancefield, R. C. and Hare, R. (1935) "The serological differentiation of pathogenic and non-pathogenic strains of hemolytic streptococci from parturient women," J Exp Med, 61 (3) 335-349.

11.Lewin, E. B. and Amstey, M. S. (1981) "Natural history of group B streptococcus colonization and its therapy during pregnancy," American Journal of Obstetric and Gynecology, 139 (5) 512-515.

12.Madani, T. A, Harding, G. K, Helewa, M. and Alfa, M. J. (1998) "Screening pregnant women for group B streptococcus colonization," Infection, 26 (5) 288-291.

13.Mandell, Douglas and Bennett. (2009) Principals and practice of infectious diseases, Churchill Livingstone, Elsevier, USA.

14.Manning, S. D, Tallman, P, Baker, C. J, Gillespie, B, Marrs, C. F. and Foxman, B. (2002) "Determinants of co-colonization with group B streptococcus among heterosexual college couples," Epidemiology, 13 (5) 533-539.

15.Manning, S. D, Neighbors, K, Tallman, P. A, Gillespie, B, Marrs, C. F, Borchardt, S. M, Baker, C. J, Pearlman, M. D. and Foxman, B. (2004) "Prevalence of group B streptococcus colonization and potential for transmission by casual contact in healthy young men and women," Clin Infect Dis, 39 (3) 380-388.

16.Newton, E. R, Butler, M. C. and Shain, R. N. (1996) "Sexual behavior and vaginal colonization by group B streptococcus among minority women," Obstet Gynecol, 88 (4 pt 1) 577-582.

17.Ramos, E, Gaudier, F. L, Hearing, L. R, Del Valle, G. O, Jenkins, S. and Briones, D. 
(1997) "Group B streptococcus colonization in pregnant diabetic women," Obstet Gynecol, 89 (2) 257-260.

18. Royal College of Obstetricians and Gynaecologists. (2012) "The prevention of early-onset neonatal group B streptococcal disease" [Online] UK: Green top guideline no 36. [Accessed July 30, 2014], Available at: http://www.rcog.org.uk/womenshealth/clinical-guidance/prevention-earlyonset-neonatal-group-b-streptococcaldisease-green-

19.Sharma, M, Eriksson, B, Marrone, G, Dhaneria, S. and Lundborg, C. S. (2012) "Antibiotic prescribing in two private sector hospitals; one teaching and one non teaching: a cross-sectional study in Ujjain, India," BMC Infectious diseases, 12 (155) 19.

20.Stoll, B. J. and Schuchat, A. (1998) "Maternal carriage of group B streptococci in developing countries," Pediatr Infect Dis J, 17 (6) 499-503.

21. Tacconelli, E, Angelis, G. D, Cataldo, M. A, Pozzi, E. and Cauda R. (2008) "Does antibiotic exposure increase the risk of methicillin-resistant staphylococcus aureus (MRSA) isolation? A systematic review and meta-analysis," Journal of antimicrobial chemotherapy, 61 (1) 26-38.
22.Tsolia, M, Psoma, M, Gavrili, S, Petrochilou, V, Michalas, S, Legakis, N. and Karpathios T. (2003) "Group B streptococcus colonization of Greek pregnant women and neonates: prevalence, risk factors and serotypes," Clin Microbiol Infect, 9 (8) 832-838.

23.Turrentine, M. A, Greisinger, A. J, Brown, K. S, Wehmanen, O. A. and Mouzoon, M. E. (2013) "Duration of intrapartum antibiotics for group B streptococcus on the diagnosis of clinical neonatal sepsis," [Online]. Infect Dis Obstet Gynecol. [Accessed July 31, 2014], Available at: http://www.ncbi.nlm.nih.gov/pubmed/23 606801

24.Uduman, S. A, Chatterjee, T. K, AlMouzan, M. I. and Al-Suleiman, S. (1985) "Group B streptococci colonization among Saudi women in labour and neonatal acquisition," International journal of Gynaecoly Obstetrics, 23 (1) 21-24.

25.Winn, H. N. (2007) "Group B streptococcus infection in pregnancy," Clinics in Perinatology, 34 (3) 387-392.

29.Zamzami, T. Y, Marzouki, A. M. and Nasrat, H. A. (2011) "Prevalence rate of group B streptococcal colonization among women in labour at King Abdul-Aziz University Hospital," Arch Gynecol Obstet, 284 (3) 677-679. 